\title{
JAB1/CSN5: A Multifunctional Protein in Cancer
}

\author{
Arata Nishimoto ${ }^{1 *}$, Naruji Kugimiya ${ }^{1}$, Tohru Hosoyama ${ }^{1}$, Tadahiko Enoki ${ }^{1}$, Tao-Sheng Li ${ }^{2}$ and Kimikazu Hamano \\ ${ }^{1}$ Department of Surgery and Clinical Science, Yamaguchi University Graduate School of Medicine, Ube, Yamaguchi 755-8505, Japan \\ ${ }^{2}$ Department of Stem Cell Biology, Nagasaki University Graduate School of Biomedical Sciences, Nagasaki 852-8523, Japan
}

\begin{abstract}
C-Jun activation domain-binding protein 1 (JAB1) was originally identified as a c-Jun co-activator and subsequently discovered to be a component of the COP9 signalosome (CSN) complex. JAB1, which is also known as CSN5, affects many partner proteins through protein-protein interaction, which leads to protein degradation and transcriptional activation. Thereby, JAB1/CSN5 functions as a multifunctional protein involved in the regulation of cell cycle, signal transduction, and DNA repair. In particular, JAB1/CSN5 plays an essential role in tumorigenesis by degrading tumor suppressor proteins and activating oncogenic transcription factors. JAB1/CSN5 overexpression has been observed in various types of cancer, and it has been multifunctionally involved in cancer progression.
\end{abstract}

In this review, we provide an overview of the roles of JAB1/CSN5 in tumorigenesis and summarize recent findings that highlight the novel roles of JAB1/CSN5 in this process.

Keywords: JAB1/CSN5; Tumorigenesis; Protein-protein interaction; Nuclear export; Protein degradation; Transcriptional activation

\section{Introduction}

Inactivation of oncogenic proteins and induction of tumor suppressor proteins have provided an effective avenue for treating cancer cells. However, targeting candidate oncogenic and tumor suppressor proteins exist so many. Therefore, the protein which positively regulates oncogenic proteins and negatively regulates tumor suppressor proteins is a potential target for novel and effective cancer therapy. In this study, we discuss c-Jun activation domain-binding protein 1 (JAB1), which has been implicated to be multifunctionally involved in tumorigenesis. JAB1 interacts with multiple proteins and affects many aspects of tumorigenesis, such as protein degradation of tumor suppressors and activation of oncogenic transcription factors. Therefore, JAB1 has the potential to be an effective target for cancer therapy.

JAB1 was originally identified as a c-Jun co-activator. JAB1 stabilizes c-Jun DNA-binding and potentiates its transcriptional activity [1]. JAB1 was subsequently discovered as the fifth integral component of the constitutive photomorphogenic-9 (COP9) signalosome (CSN) complex. For this reason, JAB1 is also referred to as CSN5 [2]. JAB1/ CSN5 has many binding partners and affects their protein stability and transcriptional activity. Thereby, JAB1/CSN5 functions as a multifunctional protein involved in the regulation of cell cycle, signal transduction, and DNA repair [3].

JAB1/CSN5 plays an essential role in tumorigenesis by functionally inactivating several key tumor suppressor proteins including p53 [4,5], Smad7 [6], Runx3 [7], and the cyclin-dependent kinase inhibitor p27 ${ }^{\text {Kipl }}$ (p27) $[8,9]$. JAB1/CSN5 translocates these proteins from the nucleus to the cytoplasm and subsequently degrades them in the proteasome. In addition, JAB1/CSN5 is a transcriptional co-activator for c-Jun [1], MYC [10], HIF-1 $\alpha$ [11,12] and STAT3 [13]. JAB1/CSN5 overexpression has been found in various types of cancer [14], and it has been multifunctionally involved in many aspects of tumorigenesis.

In this review, we provide an overview of the roles of JAB1/CSN5 in tumorigenesis and summarize recent research progress on cellular functions of JAB1/CSN5 in this process.

\section{JAB1/CSN5 as a Member of the CSN}

JAB1, which is also referred to as CSN5, is the fifth integral component of the CSN complex. Native-PAGE analyses have revealed that JAB1/CSN5 exists as a holocomplex, two smaller complexes (250 to $300 \mathrm{kDa}$ and $100 \mathrm{kDa}$ ), and monomeric form. The CSN holocomplex and one smaller complex (250 to $300 \mathrm{kDa}$ ) are primarily located in the nucleus. On the other hand, the other smaller complex $(100 \mathrm{kDa})$ and monomeric form are primarily located in the cytoplasm [9]. Furthermore, immunofluorescent staining and SDS-PAGE analyses also have revealed that JAB1/CSN5 is located in both the nucleus and the cytoplasm $[9,13]$. Interestingly, recent study has revealed that the transforming effects of JAB1/CSN5 require CSN subunits for assembly of the full COP9 signalosome and the isopeptidase activity of CSN5, which potentiates the transcriptional activity of MYC [15]. Furthermore, smaller complex-associated with JAB1/CSN5 (100 kDa), rather than the CSN holocomplex, drives p27 degradation in the cytoplasm [16]. These findings seem to be consistent with the cellular localization of the CSN holocomplex and smaller complex-associated with JAB1/ CSN5. However, the detailed function of the CSN holocomplex, smaller complexes-associated with JAB1/CSN5, and monomeric form remains unclear. Further studies are needed to investigate the roles of the CSN holocomplex, smaller complex-associated with JAB1/CSN5, and monomeric form of JAB1/CSN5 in various cellular events such as cytoplasmic translocation, protein degradation, and activation of transcription factors.

\section{JAB1/CSN5 Structure and its Structural Function}

$J A B 1 / C S N 5$ gene is highly conserved in humans, mouse, Drosophila, fission yeast, and Arabidopsis, suggesting that JAB1/ CSN5 is critical for development and survival. Indeed, JAB1/CSN5deficient mice had an embryonical lethality [17]. Human JAB1/CSN5

*Corresponding author: Arata Nishimoto, Department of Surgery and Clinica Science, Yamaguchi University Graduate School of Medicine, 1-1-1 MinamiKogushi, Ube, Yamaguchi 755-8505, Japan, Tel: +81 83622 2261; Fax: +81 836 22 2423; E-mail: anishimo@yamaguchi-u.ac.jp

Received January 11, 2014; Accepted February 18, 2014; Published February 20, 2014

Citation: Nishimoto A, Kugimiya N, Hosoyama T, Enoki T, Li TS, et al. (2014) JAB1/CSN5: A Multifunctional Protein in Cancer. J Mol Genet Med 7: 099 doi: 10.4172/1747-0862.1000099

Copyright: (c) 2014 Nishimoto A, et al. This is an open-access article distributed under the terms of the Creative Commons Attribution License, which permits unrestricted use, distribution, and reproduction in any medium, provided the original author and source are credited 
gene is located on chromosome 8q13. JAB1/CSN5 protein consists of 334 amino acids and has a molecular mass of $38 \mathrm{kDa}$. JAB1/CSN5 protein is localized in both the nucleus and the cytoplasm. JAB1/CSN5 has two structural characteristics. One is nuclear export signal (NES)like sequence, and the other is Mpr1-Pad1-N-terminal (MPN) domain containing metalloenzyme (JAMM) motif. JAB1/CSN5 directly binds to $\mathrm{p} 27$ and mediates cytoplasmic shuttling of p27 from the nucleus in a nuclear export inducer, CRM1-dependent manner through a NESlike sequence [9]. Furthermore, JAB1/CSN5 has a MPN domain that contains a JAMM motif. The JAMM motif in JAB1/CSN5 cleaves the ubiquitin-like protein, Nedd 8 from the Cull subunit and catalytically regulates the enzymatic activity of SCF E3 ubiquitin ligases [18]. Interestingly, all components of the CSN resemble to all subunits of the lid complex of the proteasome in terms of subunit composition and the amino acid sequence of each subunit $[19,20]$. Indeed, the JAB1/ CSN5 MPN domain containing a JAMM motif resembles to the amino acid sequence of $26 \mathrm{~S}$ proteasome lid component, RPN11, which is responsible for the ubiquitin cleavage from the ubiquitinated proteins in the proteasome $[18,21]$. These similarities suggest that these proteins originate from a common ancestor and share similar molecular properties. There are some evidences that the CSN interacts with the proteasome and competes with the lid of the proteasome [22,23]. Among the effects of JAB1/CSN5 on its binding partner proteins, cytoplasmic shuttling and protein degradation can be substantially explained by NES-like sequence, JAMM motif, and the similarity between the CSN holocomplex and the lid of the proteasome. Compared with such effects of JAB1/CSN5, the mechanism by which transcription factors are activated can't be substantially explained. Some studies have reported that JAB1/CSN5 is located on chromatin DNA [24,25]. However, it still remains unclear how JAB1/CSN5 exists on chromatin DNA, its binding to chromatin DNA is direct or indirect, and whether other partner proteins exist or not. Further studies are needed to elucidate the mechanism by which JAB1/CSN5 binds to chromatin DNA.

\section{Multifunction of JAB1/CSN5}

JAB1/CSN5 interacts with many proteins, and affects many activities, such as the regulation of protein degradation and stability, cytoplasmic shuttling from the nucleus, the DNA-binding activity of transcription factors, and their transcriptional activity.

\section{Regulation of protein degradation and stability}

JAB1/CSN5 promotes the degradation of its binding partner proteins including p27 [8,9], p53 [4,5], SMAD7 [6], Runx3 [7], Rad9Rad1-Hus1 (9-1-1) complex [26], MYC [10], estrogen receptor a [27] misfolded cystic fibrosis transmembrane conductance regulator (CFTR) [28], West Nile virus capsid protein [29], and endothelin type A and B receptors [30]. JAB1/CSN5 degrades various proteins localized in the nucleus, the cytoplasm, and the cell membrane through protein-protein interaction. Interestingly, JAB1/CSN5 induces nuclear export and subsequent degradation of its binding partner proteins in the nucleus (e.g. p27, p53, 9-1-1 complex, SMAD7, and Runx3). JAB1/CSN5 induces nuclear export and subsequent degradation of p27, leading to exit from G1 phase and entry into S phase [9]. JAB1/ CSN5 enhances nuclear export and cytoplasmic degradation of p53 through MDM2-mediated p53 ubiquitination [4,5]. JAB1/CSN5 also mediates translocation of 9-1-1 complex from the nucleus to the cytoplasm and subsequent degradation of 9-1-1 complex [26]. Nuclear export and subsequent degradation of p53 and 9-1-1 complex caused by JAB1/CSN5 leads to the impairment of DNA damage checkpoint. Furthermore, JAB1/CSN5 enhances nuclear export and cytoplasmic degradation of SMAD7 [6], and Runx3 [7] which function as tumor suppressors. On the other hand, JAB1/CSN5 stabilizes several JAB1/ CSN5-interacting proteins including HIF-1a [11,12], c-Jun [31], and transformed mouse 3T3 cell double minute 2 (Mdm2) [5]. JAB1/ CSN5 may determine to degrade or stabilize its interacting proteins depending on their ubiquitination status. Further studies are needed to elucidate the mechanism to determine degradation or stabilization of JAB1/CSN5-interacting proteins.

\section{Regulation of cytoplasmic shuttling from the nucleus}

JAB1/CSN5 controls nuclear export of p27, p53, 9-1-1 complex, Runx3, and SMAD7. These JAB1/CSN5-interacting proteins translocate from the nucleus to the cytoplasm, and are subsequently degraded in the proteasome. As JAB1/CSN5 contains a nuclear export signal (NES) sequence and translocates from the nucleus to the cytoplasm through the interaction with CRM1, which can transport its interacting proteins to the cytoplasm, JAB1/CSN5-interacting proteins in the nucleus may translocate to the cytoplasm as a consequence of JAB1/CSN5 translocation to the cytoplasm.

\section{Regulation of the DNA-binding activity of transcription factors and their transcriptional activity}

JAB1/CSN5 was initially identified as a co-activator of c-Jun, a member of the activating protein-1 (AP-1) complex [1]. JAB1/CSN5 can specifically stabilize c-Jun protein binding to its DNA binding site and potentiate c-Jun-induced transcriptional activity. However, JAB1/CSN5 does not affect JunB or v-Jun DNA-binding [1]. The involvement of JAB1/CSN5 as a co-activator has been demonstrated for several transcription factors in addition to c-Jun. JAB1/CSN5 is a transcriptional co-activator for heart and neural crest derivatives expressed 2 (HAND2) [32], MYC [10], and HIF-1a [11,12]. Furthermore, our finding shows that JAB1 knockdown decreased STAT3 DNA-binding activity and the expression levels of its target genes [13]. These results suggest that JAB1/CSN5 positively regulates STAT3 DNA-binding activity and the expression levels of its target genes. Recent studies have suggested that JAB1/CSN5 associates with chromatin DNA. Chromatin immunoprecipitation (ChIP) experiments showed that JAB1/CSN5 and its interactor, SMYD3 exist in the INK4a promoter [24]. Furthermore, JAB1/CSN5 as a Cockayne syndorome group A protein-binding partner was detected in the solubilized chromatin fraction in response to UV irradiation [25]. However, it still remains unclear how JAB1/CSN5 exists on chromatin DNA. Further studies to identify the protein complex-associated with JAB1/CSN5 on chromatin DNA are needed.

\section{JAB1/CSN5 in Cancer}

As described above, the functions of JAB1/CSN5 have contributed to oncogenesis. JAB1/CSN5 transports the tumor suppressor proteins including p27, p53, 9-1-1 complex, Runx3, and SMAD7 to the cytoplasm, and subsequently degrades these proteins in the proteasome. Furthermore, JAB1/CSN5 activates oncogenic transcription factors including c-Jun, MYC, HIF-1 $\alpha$, and STAT3.

JAB1/CSN5 is found to be overexpressed in a number of human tumors, and it is important to clarify whether this overexpression is sufficient in tumorigenic process. To investigate more directly how JAB1/CSN5 overexpression leads to tumorigenesis, a stable form of JAB1/CSN5 was ectopically expressed in mice. Consequently, the transgenic mice developed myeloproliferative disorders [24]. However, the tumorigenic phenotype was less severe, suggesting that JAB1/ CSN5 collaborates with other oncogenic proteins. Interestingly, JAB1/ 
CSN5 was found to act as a master regulator of wound gene expression signature in breast cancer cells in cooperation with MYC [10]. Wound gene expression signature seems to reflect the gene expression signature of metastasis and invasion. Therefore, this result suggests that JAB1/ CSN5 acquires metastatic ability to cancer cells in cooperation with MYC. JAB1/CSN5 gene is located on $8 \mathrm{q} 13$, and MYC gene is located on $8 \mathrm{q} 24$. These regions are frequently amplified in breast, prostate, and ovarian cancers [33-35]. It suggests that the amplification of 8q region leads to the acquisition of metastatic ability of cancer cells. Further studies are needed to investigate whether the combination of JAB1/ CSN5 and MYC has effects on the other phenotypes of cancer cells, such as drug resistance, cancer stemness, and cell survival. Furthermore, JAB1/CSN5 is involved in DNA repair. In JAB1/CSN5-deficient cells, RAD51 expression level was reduced by the accumulated p53 binding to the RAD51 promoter [36]. Recent studies showed that the combination of JAB1/CSN5 knockdown and cisplatin-induced DNA damage increased apoptosis compared with JAB1/CSN5 knockdown alone [37]. Furthermore, JAB1/CSN5 knockdown increased UV radiation- and ionizing radiation-induced apoptosis. In contrast, JAB1/ CSN5 overexpression blocked UV radiation-, ionizing radiation-, and cisplatin-induced apoptosis [38]. These results suggest that JAB1/CSN5 knockdown reduces RAD51 expression level, leading to the decrease in the ability to repair the DNA lesions.

\section{JAB1/CSN5 and Prognosis}

A number of studies have implicated that JAB1/CSN5 overexpression correlated with reduced p27 expression and poor prognosis [3]. Furthermore, the combination of JAB1/CSN5 overexpression and reduced p27 expression more highly correlated with poor prognosis [39]. While the connection between JAB1/CSN5 overexpression and reduced p27 expression is evident in human tumors, the correlation between JAB1/CSN5 overexpression and the activation of transcription factors, such as c-Jun, MYC, HIF-1 $\alpha$ and STAT3 remains to be validated in human specimens. These transcription factors are wellcharacterized oncogenes that have been demonstrated to promote cellular proliferation, metastasis, and drug resistance. Furthermore, these transcription factors have been proven to have prognostic value in a number of tumor types and to contribute to carcinogenesis. As the activated transcription factors translocate to the nucleus, we should focus on the expression level of nuclear JAB1/CSN5 but not total JAB1/CSN5. Further studies are needed to investigate whether the combination of nuclear JAB1/CSN5 overexpression and the activation of oncogenic transcription factor or nuclear JAB1/CSN5 overexpression alone more highly correlates with poor prognosis.

\section{Regulation of JAB1 Expression, Nuclear Localization, and its Activity}

$J A B 1 / C S N 5$ gene is located on $8 \mathrm{q} 13$, which is frequently amplified during cancer progression. DNA copy number gain of JAB1/CSN5 was confirmed in a study of hepatocellular carcinoma with amplification of $8 \mathrm{q}$ region [40]. Besides JAB1/CSN5 amplification, several proteins have been shown to regulate JAB1/CSN5 expression level. The oncogenic tyrosine kinase Bcr-Abl regulates the formation of JAB1/ CSN5 subcomplex, which is required for p27 down-regulation [16]. Furthermore, the human epidermal growth factor receptor 2 (HER2) oncogene increases $J A B 1 / C S N 5$ expression through the binding of $\beta$-catenin and transcription factor 4 (TCF-4) to the JAB1/CSN5 promoter [41]. Interestingly, epidermal growth factor (EGF) treatment increases nuclear JAB1/CSN5 expression level but not its total expression level, leading to the reduction of p27 expression level [42]. Psoriasin/ S100A7 also increases nuclear JAB1/CSN5 expression level but not its total expression level, resulting in the increase of AP-1 activity and the reduction of p27 expression level [43]. These results suggest that EGF treatment or psoriasin/S100A7 overexpression increases nuclear import of JAB1/CSN5, leading to an increase in nuclear JAB1/CSN5 activity. Further studies are needed to elucidate the mechanism by which JAB1/ CSN5 translocates into the nucleus. Recent study has revealed that $J A B 1 / C S N 5$ is transcriptionally activated through the binding of STAT3 to the JAB1/CSN5 promoter in breast cancer cells [44]. Combined with our finding that JAB1/CSN5 positively regulates STAT3 DNA-binding

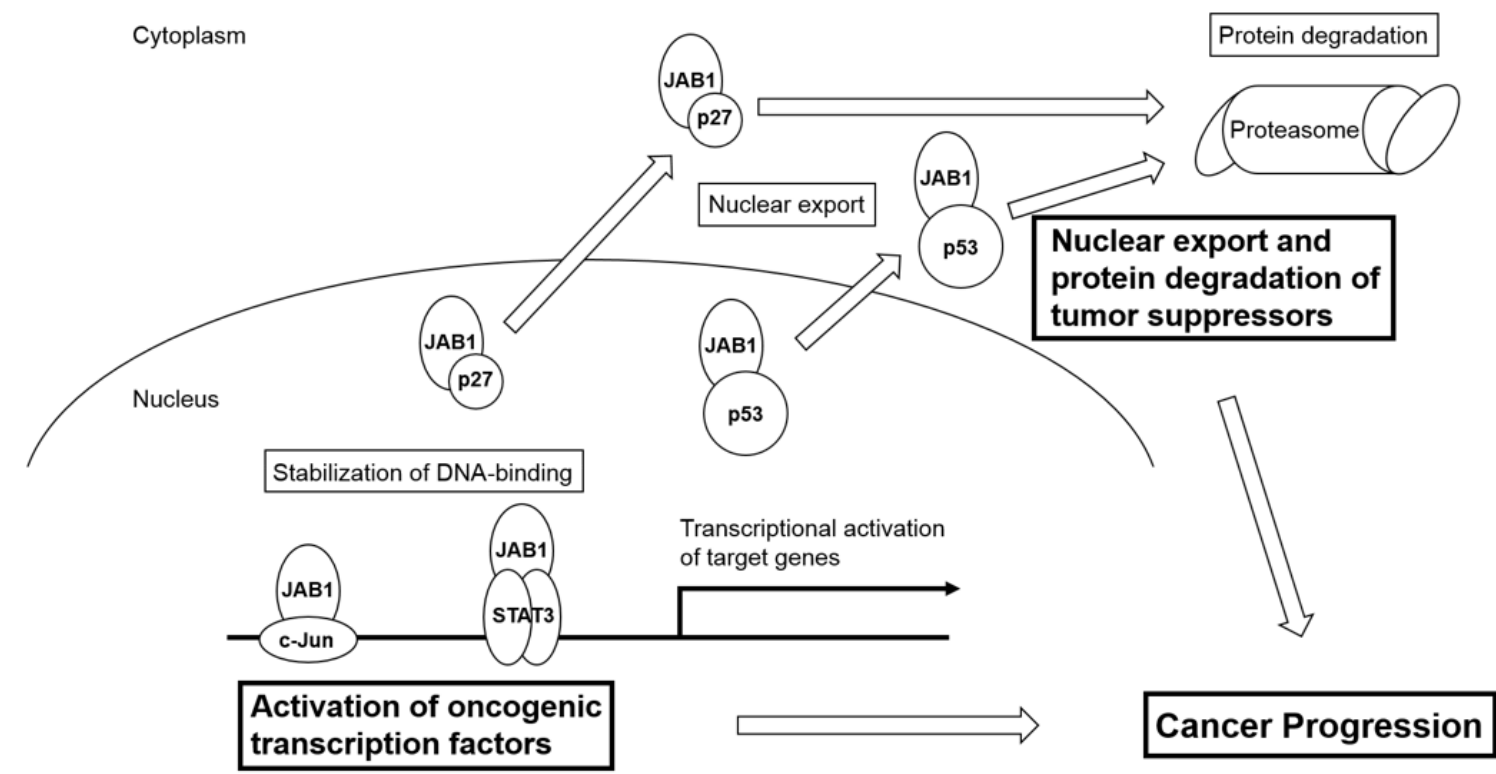

Figure 1: Overview of the roles of JAB1/CSN5 in cancer progression. In the nucleus, JAB1/CSN5 positively regulates oncogenic transcription factors through stabilization of their DNA-binding, leading to transcriptional activation of target genes. JAB1/CSN5 also negatively regulates tumor suppressor proteins through their nuclear export and protein degradation in the cytoplasm. 
activity [13], a positive feedback loop between JAB1/CSN5 and STAT3 may exist. Taken together, these studies showed a link between JAB1/ CSN5 expression and the activity of well-known oncogenes, suggesting that JAB1/CSN5 is closely involved in cancer progression.

\section{Conclusions and future Perspective}

In this review article, we provide succinct information on the roles of JAB1/CSN5 in tumorigenesis. JAB1/CSN5 interacts with multiple proteins and affects many aspects of tumorigenesis in both the nucleus and the cytoplasm. In the nucleus, JAB1/CSN5 positively regulates oncogenic transcription factors through stabilization of their DNAbinding, which leads to transcriptional activation of target genes. JAB1/ CSN5 also negatively regulates tumor suppressor proteins through their nuclear export and protein degradation in the cytoplasm (Figure 1). Consequently, JAB1/CSN5 leads to an increase in cell proliferation, cell viability, DNA repair, metastasis, and drug resistance. JAB1/CSN5 functions as a member of the CSN holocomplex, smaller complex consisting of some CSN subunits, and monomeric form. Further studies are needed to elucidate which form-associated with JAB1/CSN5 regulates protein degradation of tumor suppressors and activation of oncogenic transcription factors. Answering these unknown things will provide a novel and effective strategy for the prevention of tumor progression and lead to a successful treatment of human malignant tumors.

\section{Acknowledgements}

This work was supported by Grant-in-Aids for Young Scientific Research (B) (No. 24791425 to A. Nishimoto) from Japan Society for the Promotion of Science (JSPS) and by a grant from Takeda Science Foundation of Japan.

\section{Conflicts of interest}

The authors confirm that there are no conflicts of interest.

\section{References}

1. Claret FX, Hibi M, Dhut S, Toda T, Karin M (1996) A new group of conserved coactivators that increase the specificity of AP-1 transcription factors. Nature 383: 453-457.

2. Deng XW, Dubiel W, Wei N, Hofmann K, Mundt K, et al. (2000) Unified nomenclature for the COP9 signalosome and its subunits: an essential regulator of development. Trends Genet 16: 202-203.

3. Shackleford TJ, Claret FX (2010) JAB1/CSN5: a new player in cell cycle control and cancer. Cell Div 5: 26.

4. Oh W, Lee EW, Sung YH, Yang MR, Ghim J, et al. (2006) Jab1 induces the cytoplasmic localization and degradation of p53 in coordination with Hdm2. J Biol Chem 281: 17457-17465.

5. Zhang XC, Chen J, Su CH, Yang HY, Lee MH (2008) Roles for CSN5 in contro of p53/MDM2 activities. J Cell Biochem 103: 1219-1230.

6. Kim BC, Lee HJ, Park SH, Lee SR, Karpova TS, et al. (2004) JAB1/CSN5, a component of the COP9 signalosome, regulates transforming growth factor beta signaling by binding to Smad7 and promoting its degradation. Mol Cell Biol 24: 2251-2262.

7. Kim JH, Choi JK, Cinghu S, Jang JW, Lee YS, et al. (2009) JAB1/CSN5 induces the cytoplasmic localization and degradation of RUNX3. J Cell Biochem 107: 557-565

8. Tomoda K, Kubota Y, Kato J (1999) Degradation of the cyclin-dependentkinase inhibitor p27Kip1 is instigated by Jab1. Nature 398: 160-165.

9. Tomoda K, Kubota Y, Arata Y, Mori S, Maeda M, et al. (2002) The cytoplasmic shuttling and subsequent degradation of p27Kip1 mediated by JAB1/CSN5 and the COP9 signalosome complex. J Biol Chem 277: 2302-2310.

10. Adler AS, Lin M, Horlings H, Nuyten DS, van de Vijver MJ, et al. (2006) Genetic regulators of large-scale transcriptional signatures in cancer. Nat Genet 38 : $421-430$
11. Bae MK, Ahn MY, Jeong JW, Bae MH, Lee YM, et al. (2002) Jab1 interacts directly with HIF-1alpha and regulates its stability. J Biol Chem 277: 9-12.

12. Bemis L, Chan DA, Finkielstein CV, Qi L, Sutphin PD, et al. (2004) Distinct aerobic and hypoxic mechanisms of HIF-alpha regulation by CSN5. Genes Dev 18: $739-744$

13. Nishimoto A, Kugimiya N, Hosoyama T, Enoki T, Li TS, et al. (2013) JAB1 regulates unphosphorylated STAT3 DNA-binding activity through proteinprotein interaction in human colon cancer cells. Biochem Biophys Res Commun 438: 513-518.

14. Kato JY, Yoneda-Kato N (2009) Mammalian COP9 signalosome. Genes Cells 14: 1209-1225.

15. Adler AS, Littlepage LE, Lin M, Kawahara TL, Wong DJ, et al. (2008) CSN5 isopeptidase activity links COP9 signalosome activation to breast cancer progression. Cancer Res 68: 506-515.

16. Tomoda K, Kato JY, Tatsumi E, Takahashi T, Matsuo Y, et al. (2005) The Jab1/ COP9 signalosome subcomplex is a downstream mediator of Bcr-Abl kinase activity and facilitates cell-cycle progression. Blood 105: 775-783.

17. Tomoda K, Yoneda-Kato N, Fukumoto A, Yamanaka S, Kato JY (2004) Multiple functions of Jab1 are required for early embryonic development and growth potential in mice. J Biol Chem 279: 43013-43018.

18. Cope GA, Suh GS, Aravind L, Schwarz SE, Zipursky SL, et al. (2002) Role of predicted metalloprotease motif of JAB1/CSN5 in cleavage of Nedd8 from Cul1. Science 298: 608-611.

19. Seeger M, Kraft R, Ferrell K, Bech-Otschir D, Dumdey R, et al. (1998) A nove protein complex involved in signal transduction possessing similarities to $26 \mathrm{~S}$ proteasome subunits. FASEB J 12: 469-478.

20. Glickman MH, Rubin DM, Coux O, Wefes I, Pfeifer G, et al. (1998) A subcomplex of the proteasome regulatory particle required for ubiquitin-conjugate degradation and related to the COP9-signalosome and elF3. Cell 94: 615-623.

21. Maytal-Kivity V, Reis N, Hofmann K, Glickman MH (2002) MPN+, a putative catalytic motif found in a subset of MPN domain proteins from eukaryotes and prokaryotes, is critical for Rpn11 function. BMC Biochem 3: 28.

22. Li L, Deng XW (2003) The COP9 signalosome: an alternative lid for the $26 \mathrm{~S}$ proteasome? Trends Cell Biol 13: 507-509.

23. Peng Z, Shen Y, Feng S, Wang X, Chitteti BN, et al. (2003) Evidence for physical association of the COP9 signalosome, the proteasome, and specific SCF E3 ligases in vivo. Curr Biol 13: R504-505.

24. Mori M, Yoneda-Kato N, Yoshida A, Kato JY (2008) Stable form of JAB1 enhances proliferation and maintenance of hematopoietic progenitors. $\mathrm{J}$ Bio Chem 283: 29011-29021.

25. Groisman R, Polanowska J, Kuraoka I, Sawada J, Saijo M, et al. (2003) The ubiquitin ligase activity in the DDB2 and CSA complexes is differentially regulated by the COP9 signalosome in response to DNA damage. Cell 113 357-367.

26. Huang J, Yuan H, Lu C, Liu X, Cao X, et al. (2007) Jab1 mediates protein degradation of the Rad9-Rad1-Hus1 checkpoint complex. J Mol Biol 371: 514-527.

27. Calligé M, Kieffer I, Richard-Foy H (2005) CSN5/Jab1 is involved in liganddependent degradation of estrogen receptor $\{$ alpha\} by the proteasome. Mol Cell Biol 25: 4349-4358.

28. Tanguy G, Drévillon L, Arous N, Hasnain A, Hinzpeter A, et al. (2008) CSN5 binds to misfolded CFTR and promotes its degradation. Biochim Biophys Acta 1783: $1189-1199$

29. Oh W, Yang MR, Lee EW, Park KM, Pyo S, et al. (2006) Jab1 mediates cytoplasmic localization and degradation of West Nile virus capsid protein. $J$ Biol Chem 281: 30166-30174.

30. Nishimoto A, Lu L, Hayashi M, Nishiya T, Horinouchi T, et al. (2010) Jab1 regulates levels of endothelin type $A$ and $B$ receptors by promoting ubiquitination and degradation. Biochem Biophys Res Commun 391: 1616-1622.

31. Peth A, Berndt C, Henke W, Dubiel W (2007) Downregulation of COPg signalosome subunits differentially affects the CSN complex and target protein stability. BMC Biochem 8: 27

32. Dai YS, Hao J, Bonin C, Morikawa Y, Cserjesi P (2004) JAB1 enhances HAND2 transcriptional activity by regulating HAND2 DNA binding. J Neurosc Res 76: 613-622. 
Citation: Nishimoto A, Kugimiya N, Hosoyama T, Enoki T, Li TS, et al. (2014) JAB1/CSN5: A Multifunctional Protein in Cancer. J Mol Genet Med 7: 099 doi: 10.4172/1747-0862.1000099

33. Rummukainen J, Kytölä S, Karhu R, Farnebo F, Larsson C, et al. (2001) Aberrations of chromosome 8 in 16 breast cancer cell lines by comparative genomic hybridization, fluorescence in situ hybridization, and spectral karyotyping. Cancer Genet Cytogenet 126: 1-7.

34. Sun J, Liu W, Adams TS, Sun J, Li X, et al. (2007) DNA copy number alterations in prostate cancers: a combined analysis of published CGH studies. Prostate 67: $692-700$.

35. Dimova I, Orsetti B, Negre V, Rouge C, Ursule L, et al. (2009) Genomic markers for ovarian cancer at chromosomes 1,8 and 17 revealed by array CGH analysis. Tumori 95: 357-366.

36. Tian L, Peng G, Parant JM, Leventaki V, Drakos E, et al. (2010) Essential roles of Jab1 in cell survival, spontaneous DNA damage and DNA repair. Oncogene 29: $6125-6137$

37. Pan $Y$, Zhang Q, Tian L, Wang X, Fan X, et al. (2012) JAB1/CSN5 negatively regulates p27 and plays a role in the pathogenesis of nasopharyngeal carcinoma. Cancer Res 72: 1890-1900.

38. Pan $Y$, Zhang Q, Atsaves V, Yang H, Claret FX (2013) Suppression of JAB1/ CSN5 induces radio- and chemo-sensitivity in nasopharyngeal carcinoma through changes to the DNA damage and repair pathways. Oncogene 32: 2756-2766.
39. Sui L, Dong Y, Ohno M, Watanabe Y, Sugimoto K, et al. (2001) Jab1 expression is associated with inverse expression of p27(kip1) and poor prognosis in epithelial ovarian tumors. Clin Cancer Res 7: 4130-4135.

40. Patil MA, Gütgemann I, Zhang J, Ho C, Cheung ST, et al. (2005) Array-based comparative genomic hybridization reveals recurrent chromosomal aberrations and Jab1 as a potential target for $8 \mathrm{q}$ gain in hepatocellular carcinoma. Carcinogenesis 26: 2050-2057.

41. Hsu MC, Chang HC, Hung WC (2007) HER-2/neu transcriptionally activates Jab1 expression via the AKT/beta-catenin pathway in breast cancer cells. Endocr Relat Cancer 14: 655-667.

42. Wang J, Barnes RO, West NR, Olson M, Chu JE, et al. (2008) Jab1 is a target of EGFR signaling in ERalpha-negative breast cancer. Breast Cancer Res 10: R51.

43. Emberley ED, Niu Y, Leygue E, Tomes L, Gietz RD, et al. (2003) Psoriasin interacts with Jab1 and influences breast cancer progression. Cancer Res 63 : 1954-1961.

44. Shackleford TJ, Zhang Q, Tian L, Vu TT, Korapati AL, et al. (2011) Stat3 and CCAAT/enhancer binding protein beta (C/EBP-beta) regulate JAB1/CSN5 expression in mammary carcinoma cells. Breast Cancer Res 13: R65. 